\title{
Consumo da Alimentação Escolar e Qualidade da Dieta de Escolares
}

\section{Consumption of School Meal and Diet Quality in Schoolchildren}

\author{
Andreia Facchini ${ }^{1}$, Paula Dal Bo Campagnolo² \\ ${ }^{7}$ Nutricionista \\ ${ }^{2}$ Pós-Graduação em Nutrição e Alimentos. Universidade do Vale do Rio dos Sinos - \\ UNISINOS \\ Contato: Paula Dal Bo Campagnolo - pcampagnolo@unisinos.br
}

\begin{abstract}
Resumo
Objetivo: Verificar associação entre consumo da alimentação escolar e ingestão de nutrientes e qualidade da dieta em escolares da cidade de São Leopoldo. Métodos: Análise transversal com dados de crianças de 7 a 8 anos. Foram realizados dois recordatórios de 24 horas para a ingestão de nutrientes. 0 consumo de alimentos ultraprocessados e o Índice de Alimentação Saudável (HEI) foram avaliados a partir desse instrumento. As crianças foram questionadas quanto a origem do (s) lanche (s) realizado (s) na escola: se levado de casa, comprado na cantina ou a alimentação oferecida pela escola. Resultados: Dados de 313 crianças foram analisados e observou-se que $32,6 \%$ consumiam a alimentação escolar exclusivamente. Em comparação com os demais grupos, o que consumiu exclusivamente a alimentação escolar obteve maior consumo de fibras ( $p=0,017)$, menor consumo de ultraprocessados e menor percentual de energia vindo desse tipo de alimento $(p<0,001)$. A média da pontuação do HEI foi maior $(p=0,041)$ e a prevalência de dieta pobre foi menor $(p=0,027)$ entre os que consumiam a alimentação escolar. Conclusão: As crianças que consumiram exclusivamente a alimentação escolar apresentaram melhor qualidade da dieta, menor consumo de alimentos ultraprocessados e maior consumo de fibras e proteína em relação as que trouxeram o lanche de casa, adquiriram na cantina ou consumiram lanches de mais de uma origem.
\end{abstract}

Palavras-chave: Alimentação Escolar. Estudantes. Alimentos Industrializados.

\begin{abstract}
Objective: To investigate the association between the consumption of school meal and nutrient intake and diet quality in schoolchildren from São Leopoldo city. Methods: Cross-sectional study with data based on the children between 7-8years old. Two 24-hour dietary recalls were made for nutrient intake. The consumption of ultraprocessed foods and the Healthy Eating Index (HEI) were evaluated from this instrument. The children were asked about the origin of the school lunch (es): if taken home, bought in the canteen or the food offered by the school. Results: Data from 313 children were evaluated. 32.6\% ate school meal exclusively. Compared to other groups, the one that consumed exclusive school meal had higher fiber intake ( $p=0.017$ ), lower consumption of ultraprocessed food and a
\end{abstract}


lower percentage of energy coming from this type of food $(p<0.001)$. The average of HEl score was higher $(p=0.047)$ and the prevalence of poor diet was lower $(p=$ 0.027) among those who consumed only school meal. Conclusion: The children who ate exclusively school meal had better diet quality, lower consumption of ultraprocessed food and higher fiber intake in relation to those that brought home snack, purchased in the canteen or consumed snacks from more than one source.

Keywords: School Feeding. Students. Industrialized Foods.

\section{INTRODUÇÃO}

Na última Pesquisa de Orçamento Familiar (POF-2008-2009) realizada pelo Instituto Brasileiro de Geografia e Estatística (IBGE), pôde-se observar um crescimento significativo da prevalência de obesidade no Brasil. ${ }^{1}$ Além disso, observa-se a crescente participação de alimentos processados e ultraprocessados, que possuem baixo valor nutricional, nas refeições das famílias brasileiras. ${ }^{2-3}$ Os problemas em relação à alimentação vêm aumentando não somente no Brasil, mas em diversos países, independente de seu nível de desenvolvimento e especialmente na população infantil. , $4-8^{-8}$

Diante dessa mudança de perfil nutricional da população, faz-se necessário incentivar a promoção de hábitos saudáveis. Assim, o papel da alimentação escolar encontra-se inserido, pois possui um potencial dinâmico e eficaz na intervenção do comportamento alimentar ${ }^{9}$ e promoção de uma alimentação saudável..$^{10}$ A escola é a instituição que possui um contato contínuo e intensivo com as crianças, por isso ela é uma parte importante do ambiente social delas, podendo promover determinados comportamentos por meio de ações como a educação alimentar, oferta de alimentos, atividade física, dentre outras. Essas ações podem se perpetuar no ambiente familiar bem como ao longo do seu desenvolvimento ${ }^{11}$ e atuarem no combate a obesidade infantil. ${ }^{10}$ O Brasil possui o Programa Nacional de Alimentação Escolar (PNAE) que, ao longo dos anos, manteve um foco em melhorar as dietas das crianças em idade escolar. ${ }^{12}$ A obrigatoriedade de que $70 \%$ dos recursos financeiros sejam aplicados exclusivamente em produtos básicos e a presença do nutricionista como Responsável Técnico (RT) do programa são exemplos de ações que impactam fortemente na melhora da qualidade da alimentação oferecida às crianças. Porém, trabalhos sobre o consumo da alimentação escolar no Brasil são ainda escassos. 
Dessa forma, o objetivo desse estudo foi avaliar a qualidade da dieta dos escolares e verificar se as crianças que consomem a alimentação escolar ingerem uma dieta de melhor qualidade em comparação com as que consomem outros lanches na escola.

\section{MÉTODO}

O estudo consiste em uma análise transversal de um estudo maior, o qual consistiu em um ensaio de campo randomizado com crianças recrutadas ao nascimento no hospital Centenário, único da cidade de São Leopoldo, todas atendidas pelo Sistema Único de Saúde, entre outubro de 2001 e julho de 2002. ${ }^{13}$ As análises do presente artigo consideraram apenas os dados coletados aos 7-8 anos de idade das crianças.

O cálculo do tamanho da amostra do primeiro projeto baseou-se na frequência de aleitamento materno exclusivo até os quatro meses. Foi considerado poder de $80 \%$ e nível de confiança de $95 \%$, o que determinou um tamanho amostral de 177 crianças em cada grupo, totalizando 354 crianças. Considerando uma previsão de perdas de $25,0 \%$, foram recrutados 500 pares mãe-filho para que o número amostral fosse atingido.

\section{Dados Dietéticos}

Foram realizados dois inquéritos recordatórios de 24 horas (técnica múltiplos passos $^{14}$ ) em dias alternados (com intervalo de 2 semanas a um mês) para calcular a variação intra-individual no consumo de nutrientes. Os recordatórios foram autorelatados pelas crianças com a assistência da mãe ou responsável, o primeiro aplicado na visita domiciliar e o segundo na avaliação ambulatorial.

Para o cálculo nutricional da ingestão alimentar foi utilizado o programa Nutwin versão 1.5 ampliado com a adição de alimentos disponíveis por tabelas de composição química de alimentos e aqueles fornecidos pelas indústrias brasileiras. Foi calculado a ingestão de energia, macronutrientes, cálcio, ferro, vitamina A, vitamina $\mathrm{C}$ e fibra alimentar.

Os dois inquéritos recordatórios de 24 horas de cada criança foram avaliados de acordo com o Healthy Eating Index - HEI15 e a média da pontuação dos dois inquéritos foi utilizada nas análises. Esse índice é composto por doze componentes os quais representaram a adequação e o consumo moderado. Para os noves componentes que representaram a adequação (1-frutas totais, 2-frutas inteiras, 3verduras e legumes totais, 4- verduras verde-escuras e leguminosas, 5grãos/cereais integrais, 6-lácteos, 7-proteínas totais, 8-peixes e proteínas vegetais, 
9-ácidos graxos), a maior ingestão resultou em maior pontuação, enquanto para os três componentes que deviam ter seu consumo moderado (cereais refinados, sódio e calorias vazias), a menor ingestão resultou em maior pontuação. A pontuação total do HEI compreendeu a soma dos doze componentes e pôde atingir o máximo de 100 pontos, sendo que acima de 80 pontos foi considerada uma dieta de boa qualidade, entre as pontuações 51 e 80 classifica uma dieta que precisa melhorar, abaixo de 51 pontos foi considerada uma dieta pobre. ${ }^{15}$

Os alimentos presentes em cada um dos inquéritos recordatórios de 24 horas das crianças foram classificados de acordo com o método proposto por Monteiro et al.3 que considera a natureza, a extensão e a finalidade do processamento de alimentos, também chamada de "NOVA Classification System" por Moubarac et al. ${ }^{16}$ Para os fins deste estudo, apenas o consumo de ultraprocessados foi analisado, caracterizados como produtos alimentares formulados principalmente ou inteiramente a partir de ingredientes processados, tipicamente incluindo pouco ou nenhum alimento completo (por exemplo, pão, batatas fritas, biscoitos, doces, chocolate, refrigerantes, cereais matinais e carne processada).

A quantidade em gramas de cada alimento e o valor energético total foram calculados e a média dos dois inquéritos recordatórios de 24 horas foi utilizada nas análises.

\section{Alimentação na Escola}

As crianças foram questionadas em relação ao lanche da escola no dia anterior à entrevista e as opções de resposta foram: 1- se compraram na cantina, 2consumiram a alimentação da escola (merenda escolar), 3- levaram o lanche de casa, 4- não comeram. Foi possível marcar mais de uma opção, quando a criança consumiu dois tipos de lanches no mesmo dia (levou de casa e consumiu a alimentação escolar, por exemplo).

\section{Análise dos Dados}

A ingestão habitual de nutrientes e produtos ultraprocessados foi estimada pelo The Multiple Source Method (MSM), ${ }^{17}$ a fim de corrigir os dados dietéticos para a variabilidade intra e inter-individual (MSM, Instituto Alemão de Nutrição Humana, Alemanha).

As variáveis contínuas foram descritas por média e desvio padrão ou mediana e intervalo interquartil. As variáveis categóricas foram descritas por frequências absolutas e relativas. Os testes de associação utilizados foram o quiquadrado para 
variáveis categóricas, teste t de Student para as variáveis contínuas paramétricas e Mann-Whitney para variáveis contínuas não paramétricas. O nível de significância considerado foi de $\mathrm{p}<0,05$.

O estudo foi aprovado pelo Comitê de Ética da Universidade do Vale do Rio dos Sinos $(13 / 116,2009)$.

\section{RESULTADOS E DISCUSSÃO}

Foram avaliadas, 315 crianças, sendo que 313 frequentavam a escola e responderam a questão a respeito do consumo do lanche na escola. A média de idade foi de 7,7 anos $(\mathrm{DP}=0,7)$, sendo $43,5 \%$ do sexo feminino. A mediana de renda familiar mensal foi de 2 salários mínimos (Intervalo Interquartil $=1,9$ ) e a média de anos de estudo dos pais e das mães foi de 7,1 anos $(\mathrm{DP}=2,9)$ e 6,8 anos $(\mathrm{DP}=2,7)$, respectivamente. Foi observada menor renda familiar mensal e menor escolaridade materna entre as crianças que consumiam apenas a alimentação escolar comparado com as que consumiam outros tipos de lanches (R\$ 400,00 vs. $\mathrm{R} \$ 600,00, \mathrm{p}<0,001 ; 6,13$ anos vs. 7,17 anos, $\mathrm{p}=0,02$, respectivamente).

Entre as crianças avaliadas, foi observado que 32,6\% tinham o hábito de consumir somente a alimentação oferecida pela escola, 31,3\% levavam o lanche de casa, enquanto que 3,8\% adquiriam seu lanche na cantina da escola. A porcentagem de crianças que responderam não consumir nenhum lanche foi de $8,9 \%$, enquanto que $23,2 \%$ das crianças consumiam dois tipos de lanches (alimentação da escola mais o lanche de casa, alimentação da escola mais o lanche comprado na cantina ou lanche de casa mais o lanche comprado na cantina) (Tabela 1).

Tabela 1 - Consumo do lanche na escola no dia anterior

\begin{tabular}{lcc}
\hline & $\begin{array}{c}\mathbf{N}^{\circ} \text { de } \\
\text { crianças }\end{array}$ & $\%$ \\
\hline Merenda da escola & 102 & 32,6 \\
Lanche comprado na cantina & 12 & 3,8 \\
Lanche levado de casa & 98 & 31,3 \\
Não consumiu lanche na escola & 28 & 8,9 \\
Merenda da escola + lanche de casa & 64 & 20,4 \\
Merenda da escola + comprado na cantina & 7 & 2,2 \\
Cantina + lanche casa & 2 & 0,6 \\
\hline Total & $\mathbf{3 1 3}$ & $\mathbf{1 0 0}$ \\
\hline
\end{tabular}

O consumo alimentar das crianças está descrito na tabela 2. A média de ingestão energética diária foi de 1.569,4 Kcal e a ingestão de gordura total correspondeu, em média, a $28,6 \%$ do valor energético total consumido. Com relação à ingestão de 
alimentos ultraprocessados, os escolares consumiram, em média, 372,2 gramas ao dia, correspondendo a 776,2 kcal provenientes desses alimentos e 48,6\% do total de energia consumida. Em relação à qualidade da dieta, a pontuação média do HEI entre as crianças avaliadas foi de 65,1 , sendo que $6,8 \%$ das crianças tiveram uma alimentação considerada pobre; $88,6 \%$ uma dieta que precisa melhorar e 4,6\% uma alimentação de boa qualidade.

Tabela 2 - Características do consumo alimentar dos escolares

\begin{tabular}{lcc}
\hline & Média & Desvio Padrão \\
\hline Consumo de nutrientes & & \\
Energia (kcal) & $1.569,4$ & 380,9 \\
Carboidrato (\% do VET) & 57,5 & 7,22 \\
Proteína (\% do VET) & 14,5 & 2,9 \\
Lipídeo (\% do VET) & 28,6 & 4,8 \\
Cálcio (mg) & 537,6 & 231,6 \\
Ferro (mg) & 13,0 & 6,14 \\
Fibra (g) & 11,0 & 4,68 \\
Vitamina C (mg) & $50,1^{*}$ & $58,0^{* *}$ \\
Vitamina A (mcg) & $377,6^{*}$ & $265,6^{* *}$ \\
Consumo de alimentos ultraprocessados & \\
Gramas & 372,2 & \\
Kcal & 776,2 & 205,7 \\
\% do VET & 48,6 & 345,8 \\
HEl & & 15,2 \\
Pontuação & 65,1 & \\
\hline
\end{tabular}

VET: Valor energético total; HEl: Health Eating Index; *Mediana; ** Intervalo Interquartil.

Ao relacionar o consumo da alimentação escolar com a qualidade da dieta da criança, foi observado que o consumo de fibras e proteína foi superior entre as crianças que consumiam apenas a alimentação escolar, comparado com os demais. Em relação aos outros nutrientes avaliados, não foi observado diferença estatística significativa (Tabela 3). O consumo de ultraprocessados foi significativamente menor entre as crianças que consumiam apenas a alimentação escolar $(\mathrm{p}<0,001)$, assim como o percentual de energia vindo desse tipo de alimento $(p<0,001)$, em relação às crianças que consumiam outros tipos de lanches na escola. A média da pontuação do HEI foi de 66,6 para as crianças que consumiam apenas a alimentação escolar, enquanto que para os demais, a média de pontuação foi de 64,3 (p=0,041) (Tabela 3). A prevalência de Dieta Pobre entre os que consumiam apenas a alimentação escolar foi de 2,0\% enquanto que para os demais a taxa foi de $9,1 \%(p=0,027)$. Adicionalmente, as análises foram repetidas separadamente entre as crianças filhas de mães com menos de 8 anos de escolaridade e as filhas de mães com 8 ou mais anos de escolaridade. Essa análise foi feita com a finalidade de entender se a relação renda/escolaridade e qualidade da alimentação estaria mediando ou interferindo nos resultados. Não foi 
observada diferença nos resultados, demonstrando que a relação entre o consumo da alimentação escolar e a qualidade da dieta é independente da escolaridade materna.

Tabela 3 - Quadro comparativo entre o consumo ou não da alimentação escolar com a ingestão de nutrientes, alimentos ultraprocessados e índice da alimentação saudável (HEI)

\begin{tabular}{lccc}
\hline & $\begin{array}{c}\text { Alimentação Escolar } \\
\text { Média (DP) }\end{array}$ & $\begin{array}{c}\text { Outros } \\
\text { Média (DP) }\end{array}$ & p \\
\hline Ingestão de Nutrientes & & & \\
Energia (kcal) & $1538,2(358,1)$ & $1584,3(391,3)$ & 0,323 \\
Carboidrato (\% do VET) & $56,7(7,4)$ & $57,8(7,1)$ & 0,217 \\
Proteína (\% do VET) & $15,2(2,6)$ & $14,1(3,0)$ & 0,002 \\
Lipídeo (\% do VET) & $28,7(5,2)$ & $28,6(4,6)$ & 0,949 \\
Cálcio (mg) & $547,0(223,6)$ & $533,1(235,7)$ & 0,624 \\
Ferro (mg) & $13,9(8,1)$ & $12,6(4,9)$ & 0,073 \\
Fibra (g) & $11,9(4,3)$ & $10,5(4,8)$ & 0,017 \\
Vit C (mg) & $57,0(56,7)^{*}$ & $48,3(54,8)^{*}$ & 0,142 \\
Vit A (mcg) & $414,7(267,1)^{*}$ & $360,5(267,8)^{*}$ & 0,464 \\
\hline Consumo de alimentos ultraprocessados & & $<0,001$ \\
Gramas & $260,0(215,0)^{*}$ & $375,4(279,2)^{*}$ & $<0,001$ \\
Kcal & $641,8(346,3)^{*}$ & $768,5(442,0)^{*}$ & $<0,001$ \\
\% do VET & $43,1(12,7)$ & $51,2(16,0)$ & \\
\hline HEl & & & 0,041 \\
Pontuação & $66,6(9,2)$ & $64,3(9,2)$ & \\
\hline
\end{tabular}

DP: desvio padrão; VET: Valor energético total; HEl: Health Eating Index; *Mediana.

\section{DISCUSSÃO}

As crianças que consumiram exclusivamente a alimentação escolar apresentaram consumo maior de fibra alimentar, menor de alimentos ultraprocessados e melhor pontuação do HEI em relação às crianças que não consumiram a alimentação escolar. Adicionalmente, o consumo apenas da alimentação oferecida pela escola por parte dos alunos foi associado à menor escolaridade materna e renda familiar mensal. Considerando que, depois do consumo apenas da alimentação escolar $(32,6 \%)$, as práticas mais prevalentes foram o consumo de lanches levados de casa $(31,6 \%)$ e o consumo da alimentação da escola mais o lanche de casa $(20,4 \%)$, pode-se levantar a hipótese de que a qualidade do lanche levado de casa não seja adequada, possivelmente sendo composto por produtos ultraprocessados.

Estudo realizado com crianças do ensino fundamental da rede privada de ensino de São Paulo identificou que a composição das lancheiras, apesar de alguns aspectos positivos, mostrou-se inadequada. Houve excesso de alimentos industrializados, geralmente ricos em açúcares, gorduras e sódio e baixa presença de frutas, verduras e legumes ${ }^{18}$. A qualidade do lanche que é levado de casa para escola também reflete o hábito alimentar da família e possivelmente as demais refeições realizadas em casa. 
Ressalta-se que não houve diferença na ingestão energética total entre os alunos que consumiram apenas a alimentação da escola e os demais, sendo assim, a diferença observada foi relacionada à qualidade da dieta e não à quantidade de energia ingerida pelos escolares.

A relação encontrada entre o consumo da alimentação escolar e a condição socioeconômica da família também foi observada em estudos anteriores ${ }^{19-20}$. Esse dado pode ter relação com a disponibilidade de alimentos em casa, especialmente se tratando de uma população com baixas condições socioeconômicas, e também corrobora com estudos mais amplos, que associam as características socioeconômicas e estilo de vida com padrões dietéticos. O estudo de Olinto et al. ${ }^{21}$ verificou que entre jovens adultos de Pelotas - RS, o padrão alimentar comum brasileiro foi encontrado com maior frequência entre aqueles que tiveram menores condições econômicas, enquanto que o padrão de alimentos processados era mais provável de ser seguido por aqueles que pertenciam à classe média ou alta. Em outro estudo com crianças de escolas públicas e privadas de Viçosa - MG, Villa et al. ${ }^{22}$ apontaram que melhores condições socioeconômicas da família e maior escolaridade materna relacionou-se a com o consumo elevado de sucos artificiais e refrigerantes associados ao consumo de batata e salgados fritos, salgadinhos industrializados e macarrão instantâneo. A relação entre o consumo da alimentação escolar e escolaridade materna e renda familiar apontada em nosso estudo reforça que os padrões dietéticos são influenciados por características socioeconômicas e ambientais.

Não foi identificado registro de estudos brasileiros que tenham relacionado o consumo da alimentação escolar com a qualidade da alimentação. Nos Estados Unidos, Briefel, Wilson e Gleason ${ }^{23}$ observaram, em estudo com escolares americanos, que o consumo energético diário de alimentos com baixo valor nutricional foi menor entre as crianças que consumiam a alimentação escolar, considerando a alimentação escolar como um fator protetor para a saúde, bem como outro estudo de Kakarala, Keast e Hoerr $^{24}$ que observaram haver ingestão significativamente mais elevada de energia total e açúcar, menor ingestão de ferro, fibra dietética, e vitaminas do complexo B entre os escolares que não consumiam a alimentação escolar em comparação com os que a consumiam. Condon, Crepinsek e Fox $^{25}$ observaram que as crianças que consumiram a alimentação escolar estavam mais propensas a consumir alimentos saudáveis como leite, frutas e vegetais do que as crianças não participantes do programa. 
Estudo dessa natureza em nosso país é relevante, especialmente por conta das caraterísticas peculiares da Política de Alimentação do Escolar do país, e socioeconômicas da população estudada. Os esforços para normatizar a alimentação escolar e garantir a qualidade da mesma com ações como a da compra de alimentos da agricultura familiar tem se mostrado efetivos e devem continuar. Esses resultados reforçam o quanto é importante, para população de baixo nível socioeconômico, Políticas Públicas como essa. Porém, é importante salientar que, quando o assunto é qualidade da dieta de crianças, o caminho a percorrer ainda é longo, pois apesar do estudo ter encontrado menor consumo de ultraprocessados e melhor qualidade da dieta entre as crianças que consomem a alimentação escolar comparado às que consomem outros tipos de lanche, ainda assim, no geral da amostra, o consumo de ultraprocessados foi elevado e a pontuação do HEI baixa.

O Programa Nacional de Alimentação Escolar é um dos maiores programas de alimentação escolar do mundo, com cobertura universal e gratuidade na oferta de refeições, que inova, entre outras coisas, com a legislação de aquisições públicas, que dispensa o processo de licitação para compra da agricultura familiar. Diferentes autores vêm estudando como que os municípios do país vêm se comportando frente a essa legislação. No Rio Grande do Sul, dos 52 municípios estudados por Ferigollo et al. ${ }^{26}, 71,2 \%$ atingiram $30 \%$ de gêneros alimentícios oriundos de agricultura familiar e os produtos in natura apareceram em $92,1 \%(n=105)$ das chamadas públicas. Números semelhantes foram observados em Santa Catarina, aonde 70\% cumpriam a obrigatoriedade de investir no mínimo 30\% dos recursos advindos do Fundo Nacional de Desenvolvimento da Educação na compra da Agricultura Familiar e 48\% compravam alimentos orgânicos ${ }^{27}$.

Independente do consumo da alimentação escolar, é importante salientar que no geral, foi observada baixa qualidade na alimentação das crianças avaliadas, uma vez que $95,4 \%$ das crianças apresentaram uma dieta considerada pobre ou que necessita melhorar. O estudo também identificou que quase a metade $(48,6 \%)$ da energia diária consumida pelas crianças avaliadas provém de alimentos ultraprocessados o que pode levar ao aumento de índices lipídicos e obesidade não apenas na idade escolar, mas também se perpetuando na vida adulta. Estudos revelam que uma parcela significativa dos estudantes brasileiros apresenta elevado consumo de alimentos com alta densidade energética,4 especialmente na região sul do país ${ }^{28}$. Embora o consumo de ultraprocessados tenha sido menor entre os escolares que consomem a alimentação escolar em comparação aos demais, ainda assim é elevado, 
resultado preocupante considerando que outros estudos também vem evidenciando o aumento de consumo de alimentos processados e ultraprocessados5 e implicações especialmente no perfil lipídico de crianças e adolescentes? ${ }^{7}$

Esse estudo apresenta algumas limitações. O recordatório 24 horas é amplamente utilizado em estudos que avaliam o consumo de nutrientes porém, utilizar esse método para análises limita a observação do consumo alimentar do dia anterior apenas,29-30. Para reduzir o impacto dessa limitação e da variabilidade intraindividual, foram realizados dois recordatórios de $24 \mathrm{~h}$ com cada criança/responsável. $\mathrm{O}$ dado referente ao consumo da alimentação escolar também restringiu-se ao dia anterior à entrevista, o que representa uma limitação ao estudo, uma vez que estudos que avaliam adesão à alimentação escolar utilizam a frequência semanal de consumo,19 porém, diante da escassez de estudos que avaliam a relação entre o consumo da alimentação escolar e a qualidade da dieta em nosso meio, entende-se que os achados deste são relevantes no sentido de gerar hipóteses e incentivar novas pesquisas nesta área. Estudos futuros que avaliem de forma quantitativa e qualitativa a ingestão de alimentos da alimentação escolar, daqueles levados de casa e comprados na cantina, contribuirão para avançarmos na discussão do impacto da alimentação escolar na qualidade da dieta dos escolares e adolescentes.

No presente estudo, 32,6\% dos alunos consumiram exclusivamente a alimentação escolar, e, se considerando os que consumiram outro lanche além do oferecido pela escola, o percentual sobe para 55,2\%. Esses valores são superiores ao encontrado na Pesquisa Nacional de Saúde do Escolar - PENSE 4 , a qual identificou que 10,7\% dos estudantes da rede pública da Região Sul tinham o hábito de consumir a alimentação escolar e inferior ao estudo de Valentim et al..$^{19}$ em escolas de uma cidade do Paraná, em que a prevalência de adesão (ao menos uma vez na semana) à alimentação escolar foi $57,5 \%$. A comparação deve ser feita com cautela e considerando as diferentes formas utilizadas para considerar adesão, mas de qualquer forma, pode ser considerada distante de atender a universalidade do Programa Nacional de Alimentação Escolar (PNAE).

\section{CONCLUSÃO}

O consumo exclusivo da alimentação escolar durante o lanche da escola foi associado ao maior consumo de fibra alimentar e proteína, menor consumo de alimentos ultraprocessados e melhor pontuação do HEI. Esses resultados evidenciam a 
importância de políticas públicas como o PNAE, mas também alertam para a necessidade de outras ações a nível de saúde pública que contribuam para a redução do consumo de alimentos ultraprocessados e para o aumento do acesso dessa população à alimentos in natura.

\section{REFERÊNCIAS}

1. Instituto Brasileiro de Geografia e Estatística. Pesquisa de orçamentos familiares 2008-2009: antropometria e estado nutricional de crianças, adolescentes e adultos no Brasil. Rio de Janeiro: IBGE; 2010.

2. Monteiro CA, Levy RB, Claro RM, Castro IR, Cannon G. Increasing consumption of ultra-processed foods and likely impact on human health: evidence from Brazil. Public Health Nutr. 2010;14(1):5-13.

3. Monteiro CA, Cannon G, Levy RB, Claro RM, Moubarac J-C. The Food System. Ultra-processing. The big issue for nutrition, disease, health, well-being. World Nutr. 2012;3(12):527-69.

4. Instituto Brasileiro de Geografia e Estatística. Pesquisa nacional de saúde do escolar 2012. Rio de Janeiro: IBGE; 2013.

5. Louzada MLC, Martins APB, Canella DS, Baraldi LG, Levy RB, Claro RM, et al. Alimentos ultraprocessados e perfil nutricional da dieta no Brasil. Rev Saúde Pública. 2015; 49:1-11.

6. Momm N, Höfelmann DA. Diet quality and associated factors in children enrolled in a municipal school of Itajaí, Santa Catarina. Cad Saúde Colet. 2014; 22(1):32-9.

7. Rauber F, Campagnolo PD, Hoffman DJ, Vitolo MR. Consumption of ultraprocessed food products and its effects on children's lipid profiles: A longitudinal study. Nutr Metab Cardiovasc Dis. 2015;25(1):116-22.

8. Leal KK, Schneider BC, França GVA, Gigante DP, Santos I, Assunção MCF. Qualidade da dieta de pré-escolares de 2 a 5 anos residentes na área urbana da cidade de Pelotas, RS. Rev Paul Pediatr. 2015;33(3):310-7.

9. Ashe LM, Sonnino R. At the crossroads: new paradigms of food security,public health nutrition and school food. Public Health Nutr. 2012;16(6):1020-7.

10. Fung C, McIsaac JL, Kuhle S, Kirk SF, Veugelers PJ. The impact of a populationlevel school food and nutrition policy on dietary intake and body weights of Canadian children. Prev Med. 2013;57(6):934-40.

11. Fox MK, Dodd AH, Wilson A, Gleason PM. Association between School Food Environment and Practices and Body Mass Index of US Public School Children. J Am Diet Assoc. 2009;109(2 Suppl):S108-17.

12. Sidaner E, Balaban D, Burlany L. The Brazilian school feeding programme: an example of an integrated programme in support of food and nutrition security. Public Health Nutr. 2012;16(6):989-94.

13. Vitolo MR, Bortolini GA, Feldens CA, Drachler ML. Impacts of the 10 Steps to Healthy Feeding in Infants: a randomized field trial. Cad Saúde Publica. 2005;21(5):1448-57. 
14. Johnson RK, Soultanakis RP, Matthews DE. Literacy and body fatness are associated with underreporting of energy intake in US low income women using the multiple-pass 24-hours recall: a doubly labeled water study. J Am Diet Assoc. 1998;98(10):1136-40.

15. Kennedy ET, Ohls J, Carlson S, Fleming K. The Healthy Eating Index: Design and Applications. J Am Diet Assoc. 1995;95(10):1103-8.

16. Moubarac JC, Parra DC, Cannon G, Monteiro CA. Food classification systems based on food processing: significance and implications for policies and actions: a systematic literature review and assessment. Curr Obes Rep. 2014;3(2):256-72.

17. Harttig U, Haubrock J, Knüppel S, Boeing H; EFCOVAL Consortium. The MSM program: web-based statistics package for estimating usual dietary intake using the multiple source method. Eur J Clin Nutr. 2011;65 (Suppl 1):S87-91.

18. Matuk TT, Stancari PC, Bueno MB, Zaccarelli EM. Composição de lancheiras de alunos de escolas particulares de São Paulo. Rev Paul Pediatr. 2011;29(2):157-63.

19. Valentim EA, Almeida CCB, Taconeli CA, Osório MM, Schmidt ST. Fatores associados à adesão à alimentação escolar por adolescentes de escolas públicas estaduais de Colombo, Paraná, Brasil. Cad Saúde Pública. 2017; 33(10):1-9.

20. Sturion GL, Silva MV, Ometto AH, Furtuoso MO, Pipitone MP. Fatores condicionantes da adesão dos alunos ao Programa de Alimentação Escolar no Brasil. Rev Nutr. 2005;18(2):167-81.

21. Olinto MT, Willett WC, Gigante DP, Victora CG. Sociodemographic and lifestyle characteristics in relation to dietary patterns among young Brazilian adults. Public Health Nutr. 2010;14(1):150-9.

22. Villa JKD, Silva AR, Santos TSS, Ribeiro AQ, Pessoa MC, Sant'Ana LFR. Padrões alimentares de crianças e determinantes socioeconômicos, comportamentais e maternos. Rev Paul Pediatr. 2015;33(3):302-9.

23. Briefel RR, Wilson A, Gleason P. Consumption of low-nutrient, energy-dense foods and beverages at school, home, and other locations among school lunch participants and nonparticipants. J Am Diet Assoc. 2009;109(2 Suppl):S7990.MB

24. Kakarala M, Keast DR, Hoerr S. Schoolchildren's Consumption of Competitive Foods and Beverages, Excluding à la Carte. J Sch Health. 2010;80(9):429-35.

25. Condon EM, Crepinsek MK, Fox MK. School Meals: types of foods offered to and consumed by children at lunch and breakfast. J Am Diet Assoc. 2009;109(2 Suppl):S67-78.

26. Ferigollo D, Ramos Kirsten V, Heckler D, Torres Figueredo AO, Perez-Cassarino J, Triches RM. Aquisição de produtos da agricultura familiar para alimentação escolar em municípios do Rio Grande do Sul. Rev Saúde Pública. 2017;51:1-10

27. Castellani ALA, Trentini T, Nishida W, Rossi CE, Costa LCF, Vasconcelos FAG. Purchase of family farm and organic foods by the Brazilian School Food Program in Santa Catarina state, Brazil. Rev. Nutr. 2017;30(5):651-62.

28. Azeredo CM, Rezende LF, Canella DS, Moreira Claro R, Castro IR, Luiz OC, et al. Dietary intake of Brazilian adolescents. Public Health Nutr. 2014;18(7):1215-24. 
29. Assumpção D, Barros MBA, Fisberg RM, Carandina L, Goldbaum M, Cesar CLG. Qualidade da dieta de adolescentes: estudo de base populacional em Campinas, SP. Rev Bras Epidemiol. 2012;3(15): 605-16.

30. Conceição SIO, Santos CJN, Silva AAM, Silva JS, Oliveira TC. Consumo alimentar de escolares das redes pública e privada de ensino em São Luís, Maranhão. Rev Nutr. 2010;23(6):993-1004.

Submissão: $21 / 04 / 2019$

Aprovação: 16/07/2020 